International Journal of Engineering \& Technology, $7(2.31)(2018) 94-96$
International Journal of Engineering \& Technology
WPC
Website: www.sciencepubco.com/index.php/IJET
Research paper

\title{
Methods to improve electro kinetic energy conversion efficiency in nanoscale channel
}

\author{
Manu P Nair ${ }^{*}$, Jeetu S Babu ${ }^{2}$ \\ ${ }^{1}$ Department of Mechanical Engineering, Amrita Vishwa Vidyapeetham, Amritapuri, India. \\ ${ }^{2}$ Department of Mechanical Engineering, Amrita Vishwa Vidyapeetham, Amritapuri, India.
}

\begin{abstract}
With the help of MD simulation in a Lennard jones system, Argon fluid flowing through a Platinum channel, we have demonstrated a practical approach to tune slip length in nanoscale flows. It was found that the presence of solid obstacles in the channel can manipulate the slip length similar to the variation of slip length with respect to fluid solid interaction potential. These results can act as a guidance to improve the electro kinetic energy conversion efficiency in nanoscale flows which will have immense application in the development of nanofluidic battery.
\end{abstract}

\section{Introduction}

Nanofluidic batteries are fascinating energy generation systems for converting mechanical work into electrical power. Due to its simplicity, harvesting electrical power with this nanofluidic battery system has gained considerable attention. When a flow of an aqueous solution is induced inside a nanochannel whose walls are charged a streaming current and electric potential will be produced. [1 ,2 ]. This is how energy conversion happens inside nanofluidic battery. One of the challenges of the nanofluidic battery system is its low energy conversion efficiency. A maximum of 5\% energy conversion efficiency was reported for track-etched polyethylene terephthalate (PET) nanopores. [3]

Studies have shown that a nanochannel with hydrodynamic slip, could be a viable method to improve the efficiency of nanofluidic batteries. Increase in the hydrodynamic slip length will reduce the viscous dissipation thereby reducing the dissipation loss. At nanoscale dimensions inside a nanochannel the surface area to volume ratio will be high, so the solid-fluid interaction at interface is one of the major factor which influences the hydrodynamic slip length. Recent studies in literature have pointed out that a complex interplay exists between the interfacial slip and the energy conversion mechanism which may lead to a reduction in the expected enhancement.

Heyden et.al conducted experimental study on variation of energy conversion efficient in a Nano fluidic $\mathrm{KCl}$ battery with respect to channel width and ion concentration, maximum efficiency was observed at $75 \mathrm{~nm}$ channel width, which is due to the overlapping of double layers [4]. Xie et.al performed experimental study in single track etched non-pores for determining the trend of energy conversion efficiency with respect to streaming current and conductance. Their results show that for higher surface charge density will gives higher energy conversion efficiency [5]. Bonthius et.al compared water-nano tube model using continuum and molecular dynamics simulation and found that slip length has a positive impact on the energy conversion efficiency [6]. Daigugji et.al found by computational fluid dynamics that for a electochemomechanical Nano fluidic battery, maximum energy conversion efficiency is when the channel width is approximately double the debye length of the solution [5]. But, studies have shown that the pore surface modifications in the nanofluidic batteries affect the slip behavior [7]. A complex coupling is found to exist between the underlying electrostatics and hydrodynamics over interfacial scales having a direct implication on the energy conversion efficiency in such systems [8].

In the present work, we concentrate on the effect of nanochannel width and presence of obstacle on the hydrodynamic slip length and to determine the possibilities of tuning the hydrodynamic slip length with the help of molecular dynamics (MD) simulation. In the subsequent section, we discuss the computational methodology used $\&$ in the later section the major results obtained are discussed.

\section{Methodology}

Our present work is done using molecular dynamics simulation with the aid of open source LAMMPS package [9]. The results can be visualized using visual molecular dynamics software OVITO [10,11]. MD is governed by classical laws, most importantly Newtons Second law, $\mathrm{F}=\mathrm{M} \mathrm{x}$ a, where $\mathrm{F}$ is the net force,$M$ equals to mass and $a$ is the acceleration. It uses verlet algorithm ie; for a given initial set of position and velocities the subsequent time evolution is completely determined so that the entire trajectory of atoms could be obtained. 


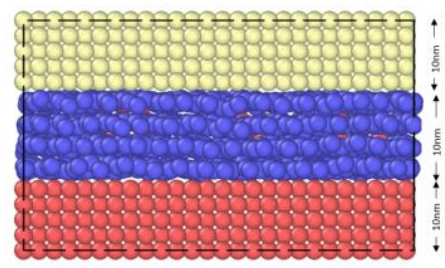

Fig. 1: Simulation domain

Table 1: Lennard Jones Parameters Used

\begin{tabular}{lll}
\hline & $\boldsymbol{\varepsilon}(\mathbf{k c a l} / \mathbf{m o l e})$ & $\boldsymbol{\sigma}\left(\mathbf{A}^{\mathbf{o}}\right)$ \\
\hline Wall-wall & 0.120183 & 2.475 \\
Fluid-fluid & 0.238 & 3.04 \\
Wall-fluid & 0.042281 & 2.7575
\end{tabular}

The simulation system consists of Argon- Platinum system with Platinum channel, Argon Fluid \& a platinum obstacle. Simple LJ potential interaction is used to simulate the system. Atoms consist of closely packed FCC structure.

The slip length is determined by using equilibrium MD - Green kubo relations [12].The effects of various parameters like channel width and number of obstacles present is investigated to understand the effect of these parameters on the slip length. Figure 1 shows the simulation domain \& Table 1 shows the parameters used for the inter atomic potential. The simulation was run for 12 lakh steps with a time step of 1.0 femtoseconds using velocity verlet integration algorithm.

\section{Results}

In this study, we have investigated the effect of varying the fluid solid interaction, channel width and the number of obstacles present in the nanochannel. Figure 2 shows the variation of slip length in a channel of width $10 \mathrm{~nm}$ with respect to $\mathrm{Ar}-\mathrm{Pt}$ interaction potential. As the wall- fluid interaction energy reduces the slip length is found to increase, this is because as the interaction reduces the fluid become more phobic and as a result the flow rate will increase. This is similar to the result obtained by Mohammed \& Babu[13].

The manipulation of slip length by varying the interaction potential is a computational methodology. This can be practically implemented by different methods. In this paper, we have demonstrated this by adding solid obstacle in the flow field, Similar to modelling nanofluidic flow through the channel. Figure 3 shows the variation of slip length in a channel, of width (d) $10 \mathrm{~nm} \& 15 \mathrm{~nm}$, with respect to the number of obstacle of diameter $5 \mathrm{~nm}$. It is seen that as the number of obstacle increases there is a reduction in slip length.

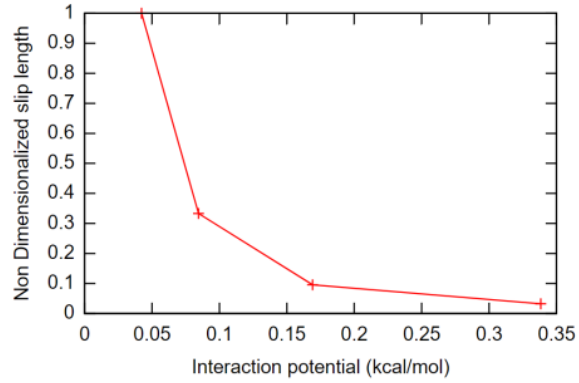

Fig. 2: Variation of Slip length in a channel of width $10 \mathrm{~nm}$ with respect to Ar-Pt interaction Potential

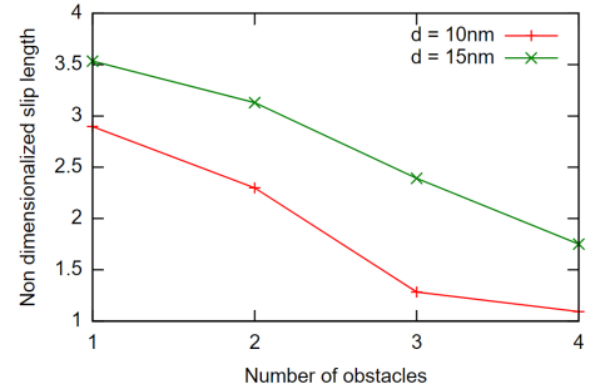

Fig. 3: Variation of slip length in a channel of width (a) d = 10nm \& (b) d $=15 \mathrm{~nm}$ with respect to the number of obstacles with diameter $5 \mathrm{~nm}$

When we compare the variation with respect to the channel width, there is a reduction in the slip length with decreasing channel diameter. These results show a practical method to tune slip length and thus guideline to improve the electro kinetic energy conversion efficiency.

\section{Conclusion}

With the help of MD simulations in a Lennard jones system, Argon fluid flowing through a platinum channel, we have demonstrated a practical approach to tune slip length in nanoscale flows. It was found that the presence of solid obstacles in the channel can manipulate the slip length like the variation of slip length with respect to fluid solid interaction potential. These results can act as a guidance to improve the electro kinetic energy conversion efficiency in nanoscale flows which will have immense application in the development of nanofluidic batteries.

\section{Acknowledgment}

The authors would like to thank Science and Engineering Research Board, Department of Science and Technology, New Delhi, India, for providing us with the financial support for conducting this project (Project No. ECR/2016/001932 dated 16th February 2017).

\section{References}

[1] Yan Y, Sheng Q, Wang C, Xue J \& Chang HC, "Energy conversion efficiency of nanofluidic bateries: Hydrodynamic slip and access resistance", The Journal of Physical Chemistry C, (2003).

[2] Yang J, Lu F, Kostiuk LW \& Kwok DY, "Electrokinetic microchannel battery by means of electrokinetic and microfluidic phenomena", J. Micromech. Microeng, (2003).

[3] Ren Y \& Stein D, "Slip-enhanced electrokinetic energy conversion in nanofluidic channels", Nanotechnology, (2008).

[4] van der Heyden FHJ, Bonthius DJ, Stein D, Meyer C \& Dekker $\mathrm{C}$, "Power generation by pressure-driven transport of ions in nanofluidic channels", Nano Letters, (2007).

[5] Xie Y, Wang X, Xue J, Jin K, Chen L \& Wang Y, "Electric energy generation in single track-etched nanopores", Applied Physics Letters, (2008).

[6] Bonthius DJ., Rinne KF, Falk K, Kaplan CN, Horinek D, Berker, AN, Bocquet L \& Netz RR, "Theory and simulations of water flow through carbon nanotubes: prospects and pitfalls", journal of Physics: Condensed Matter, (2011).

[7] Daiguji H, Yang P, Szeri AJ \& Majumdar A, "Electrochemo mechanical energy conversion in nanofluidic channels", Nano letters, Vol.4, No.12,(2004), pp.2315-2321.

[8] Bakli C \& Chakraborthy S, "Electrokinetic energy conversion in nanofluidic channels: addressing loose ends in nanodevice efficiency", Electrophoresis,(2015)

[9] Plimpton SJ, "Fast Parallel Algorithms for Short-Range Molecular Dynamics", Journal of Computational Physics, (1995).

[10] Humphrey W, Dalke A \& Schulten K, "VMD-Visual Molecular Dynamics", J. Molec. Graphics, (1996) 
[11] Stukowski A, "Visualization and analysis of atomistic simulation data with OVITO-the Open Visualization Tool", Modelling Simul. Mater. Sci. Eng.,(2010).

[12] Kannam SK, Todd BD, Hansen JS \& Daivis PJ, "Slip flow in graphene nanochannels", J Chem Phys, Vol.135, (2011).

[13] Mohammed A \& Babu JS, "Molecular Dynamics Study of Fluid Solid Interfacial Slip and Its Effect on Aerodynamic Drag", MATEC Web of Conferences, (2017). 